\title{
DEVELOPMENTS ASSOCIATED WITH A BOUNDARY PROBLEM NOT LINEAR IN THE PARAMETER*
}

BY

\author{
RUDOLPH E. LANGER
}

\section{INTRODUCTION}

The expansion of a more or less arbitrary function into a series whose terms are the characteristic functions of a differential equation containing a parameter and subject to certain boundary conditions is a problem which has long claimed the attention of mathematicians.

The oldest of such expansions is the classical Fourier's series, of which the terms satisfy the differential system

$$
\begin{gathered}
u^{\prime \prime}(x)+\lambda u(x)=0, \\
u(a)=u(b), \\
u^{\prime}(a)=u^{\prime}(b),
\end{gathered}
$$

although the system

$$
\begin{gathered}
\frac{d}{d x}\left(k \frac{d u}{d x}\right)+(\lambda g-l) u=0, \\
\boldsymbol{\alpha}_{1} u(a)+\boldsymbol{\alpha}_{2} u^{\prime}(a)=0, \\
\boldsymbol{\beta}_{1} u(b)+\boldsymbol{\beta}_{2} u^{\prime}(b)=0,
\end{gathered}
$$

was used as a source of characteristic functions as early as 1835 . Liouville, $\nmid$ using the results of Sturm, was the first to investigate this latter type of development, and with varying hypotheses regarding the coefficients $k, g$, and $l$, his work has been carried on by many others, so that with suitable restrictions on these coefficients a fairly complete theory of the expansions has been evolved.

In 1908 Birkhoff $\ddagger$ broke away from the system of Sturm and Liouville by raising the order of the differential equation from 2 to $n$ and omitting the

* Presented to the Society, February 25, 1922.

f Journal de Mathématiques, vol. 1 (1836).

$\ddagger$ These Transactions, vol. 9 (1908), p. 373. 
condition that it be self adjoint.* The system whose solutions form the terms of his developments may accordingly be written

$$
\begin{gathered}
\frac{d^{n} u}{d x^{n}}+p_{1} \frac{d^{n-1} u}{d x^{n-1}}+\cdots+p_{n-1} \frac{d u}{d x}+\left(p_{n}+\lambda g\right) u=0 \\
W_{1}(u)=0 \\
\cdot \cdot \cdot \cdot \cdot \cdot \\
\cdot \cdot \cdot \cdot \cdot \\
W_{n}(u)=0
\end{gathered}
$$

the $W$ 's representing linear homogeneous expressions in $u$ and its first $(n-1)$ derivatives taken at the points $a$ and $b$.

In several cases the boundary conditions have been changed from the forms here indicated, but only a single further modification of the differential equation appears to have been made. I refer to the equations and conditions

and

$$
\begin{gathered}
p(x) u^{\prime}+(q+\hat{\lambda}) u=K(x), \\
u(0)=\gamma, \quad u(1)=\Gamma,
\end{gathered}
$$

$$
\begin{gathered}
-\frac{d}{d x}\left(p u^{\prime}\right)+\left(q+\lambda^{2}\right) u=K(x), \\
u(0)=\gamma_{1}, \quad u^{\prime}(0)=\gamma_{2}, \\
h_{1} u^{\prime}(1)-h_{2} u(1)=\gamma,
\end{gathered}
$$

employed by Hilb in 1911. $†$

Apparently, therefore, the differential equations hitherto used for this purpose are all reducible to a system of first order equations of the type

$$
a_{i} u_{i}^{\prime}=L_{i}+\lambda M_{i} \quad(i=1,2, \cdots, n)
$$

each $L_{i}$ and $M_{i}$ representing a linear expression in $u_{1}, u_{2}, \cdots, u_{n}+\neq$ They have in common, moreover, the characteristics

$$
M_{i} \equiv 0 \text { if } i \neq n, \quad M_{i} \neq=0 \text { if } i=n . \quad(i=1,2, \cdots, n)
$$

* In this connection see also: Hilbert, Grundzüge einer allgemeinen Theorie der linearen Integralgleichungen, $\mathrm{Nachrichten}$ der Gesellschaft der Wissenschaften zu Göttingen, 1904, 1905, 1906; Westfall, Zur Theorie der Integralgleichungen, Dissertation, Göttingen, 1905; Bounitsky, Sur la fonction de Green, Jo urnal de Mathématiques, 1909: † Journal für Mathematik, vol. 140, p. 205.

† See also Schur, Zur Entwicklung willkürlicher Funktionen, Math emati s che Annalen, vol. 82 (1921), p. 213; Carmichael, Boundary value and expansion problems, American Journal of Mathematics, vol. 43 (1921), p. 69. 
It is the purpose of this paper on the other hand to consider the expansion problem associated with an equation which when reduced to a system of the form (1) is characterized by the facts that

$$
\begin{gathered}
a_{i} \equiv 0 \text { if } i \neq n, \quad a_{i} \neq 0 \text { if } i=n, \\
M_{i} \neq 0
\end{gathered} \quad(i=1,2, \cdots, n) .
$$

The equation in question with boundary condition is of the form

$$
\begin{aligned}
P(\lambda) u^{\prime}(x) & =Q(\lambda, x) u(x), \\
u(a) & =h u(b),
\end{aligned}
$$

$h$ being a detinite constant (specified below), while $P$ and $Q$ are polynomials in the complex parameter $\lambda$, the coefficients of $P$ being constants while those of $Q$ are functions of $x$ which satisfy certain conditions to be specified below. It will furthermore be assumed that the poles of the rational function $Q(\lambda, x) / P(\lambda)$ are all simple.*

\section{THE FORMAL EXPANSION}

Under the assumption already made, there is no further loss of generality in assuming the degree of $P(\lambda)$ to be at least as high as that of $Q(\lambda, x)$. $\dagger$ Accordingly we have the expression in partial fractions

$$
\frac{Q(\lambda, x)}{P(\lambda)}=a_{0}(x)+\sum_{j=1}^{n} \frac{a_{j}(x)}{\lambda-\alpha_{j}} .
$$

If, now, $h$ is chosen as $h=e^{-\int_{a}^{b} a_{0}(x) d x}$, while the dependent variable is changed by the substitution

$$
u(x)=e^{\int_{a}^{x} a_{0}(x) d x} \bar{u}(x),
$$

* Observe that the system

$$
\begin{aligned}
P(\lambda) \bar{u}^{\prime}(x) & =Q(\lambda, x) \bar{u}(x), \\
\bar{u}(a) & =h_{1} e^{-\frac{q(\lambda)}{p(\lambda)}} \bar{u}(b),
\end{aligned}
$$

where $q$ and $p$ are polynomials with constant coefficients, while $q(\lambda) / p(\lambda)$ has only simple poles, is not more general than system (1) since it may be reduced to (1) by the substitution $u=\bar{u} e^{\frac{1(\lambda) x}{(a-b) p(\lambda)}}$.

+ The degree of $Q$ can exceed that of $P$ by at most one, while if $Q$ is of higher degree than $P$ the change of parameter $\lambda=\frac{1}{\bar{\lambda}}+\beta$, where $(\lambda-\beta)$ is not a factor of $P(\lambda)$, may be made and will raise the degree of $P$ to that of $Q$. 
the system takes the form

$$
\begin{gathered}
u^{\prime}(x)=\sum_{j=1}^{n} \frac{a_{j}(x)}{\lambda-\alpha_{j}} u(x), \\
u(a)=u(b) .
\end{gathered}
$$

This is the form which will be employed hereafter, and it will be assumed that the coefficients of $Q(\lambda, x)$ are such that each of the functions $a_{j}(x)$ $(j=1,2, \cdots, n)$ in (2) is real, maintains the same sign, and is integrable and bounded throughout a closed interval $a \leqq x \leqq b$.

As a matter of notation the capital will be used to indicate the integral from $a$ to $x$ of the corresponding small letter; thus

$$
\boldsymbol{\Omega}(x)=\int_{a}^{x} \omega(x) d x .
$$

Accordingly the solution of equation (2) may be written

$$
u(x)=e^{\sum_{j=1}^{n} \frac{A_{j}(x)}{\lambda-\alpha_{j}}, *}
$$

and the characteristic values of $\lambda$ for which this solution satisfies the boundary conditions are found to be the roots of the equations

$$
\sum_{j=1}^{n} \frac{A_{j}(b)}{\lambda-\alpha_{j}}=2 k \pi i
$$

$k$ being any integer or zero.

It will be assumed throughout the discussion unless the contrary is explicitly stated that equations (3) have no multiple roots, a condition which is always fulfilled when none of the roots of the equation

$$
\sum_{j=1}^{n} \frac{A_{j}(b)}{\left(\lambda-\alpha_{j}\right)^{2}}=0
$$

are characteristic values.t

Equations (3) yield, therefore, $n$ characteristic values $\lambda_{k_{1}}, \lambda_{k_{n}}, \cdots, \lambda_{k_{n}}$, corresponding to each choice of $k$. We shall return subsequently to a discussion of these values and will show that they cluster about the points $\alpha_{1}, \alpha_{2}, \cdots, \alpha_{n}$,

* The arbitrary constant factor will be omitted.

f In case two or more characteristic values do coincide the corresponding terms of the expansion must be otherwise computed (see note on p.15). 
for $|k|$ large, a sufficiently small neighborhood of $\alpha_{\nu}$ containing just one characteristic value $\lambda_{k_{\nu}}$, for each integer $k$.

Permitting $\lambda$ to take on characteristic values in the equations of system (2) and of the adjoint system

$$
\begin{aligned}
v^{\prime}(x) & =-\sum_{j=1}^{n} \frac{a_{j}(x)}{\lambda-\alpha_{j}} v(x),{ }^{*} \\
v(a) & =v(b),
\end{aligned}
$$

we have

$$
\begin{aligned}
& u_{l_{\mu}}^{\prime}(x)=\sum_{j=1}^{n} \frac{a_{j}(x)}{\lambda_{l_{\mu}}-\alpha_{j}} u_{l_{\mu}}(x), \\
& v_{k_{\nu}}^{\prime}(x)=-\sum_{j=1}^{n} \frac{a_{j}(x)}{\lambda_{k_{\nu}}-\alpha_{j}} v_{k_{\nu}}(x) .
\end{aligned}
$$

If, now, these equations are multiplied by $v_{k_{\nu}}(x)$ and $u \iota_{\mu}(x)$ respectively and are then added, it is found that

$$
\begin{aligned}
\frac{d}{d x}\left(u_{l_{\mu}} v_{k_{\nu}}\right) & =\sum_{j=1}^{n} a_{j}(x)\left[\frac{1}{\lambda_{l_{\mu}}-\alpha_{j}}-\frac{1}{\hat{\lambda}_{k_{\nu}}-\alpha_{j}}\right] u_{l_{\mu}} v_{k_{\nu}} \\
& =\left(\lambda_{k_{\nu}}-\lambda_{l_{\mu}}\right) \sum_{j=1}^{n} \frac{a_{j}(x)}{\left(\lambda_{l_{\mu}}-\alpha_{j}\right)\left(\lambda_{k_{\nu}}-\alpha_{j}\right)} u_{l_{\mu}} v_{k_{\nu}} .
\end{aligned}
$$

The quantity $u_{l_{\mu}} v_{k_{\nu}}$, however, takes on identical values at the boundary points $a$ and $b$, whence integration between these limits yields the fact that

$$
\int_{a}^{b} \sum_{j=1}^{n} \frac{a_{j}(x)}{\left(\lambda_{l_{\mu}}-\boldsymbol{a}_{j}\right)\left(\lambda_{k_{\nu}}-\alpha_{j}\right)} u_{l_{\mu}} v_{k_{\nu}} d x=0
$$

unless $l=k$ and $\mu=\nu$. We proceed to apply the relation thus established.

Let $f_{1}(x), f_{2}(x), \cdots, f_{n}(x)$, be $n$ arbitrary functions, and let us assume the possibility of expanding these functions simultaneously in series of the form

$$
f_{j}(x)=\sum_{\substack{l=-\infty \\ \mu=1,2, \ldots, n}}^{+\infty} c_{l_{\mu}} \frac{u_{l_{\mu}}(x)}{\lambda_{l_{\mu}}-\alpha_{j}} \quad(j=1,2, \cdots, n) .
$$

* The characteristic values of systems (2) and (4) are identical. 
Multiplying by $a_{j}(x) v_{k_{\nu}}(x) /\left(\lambda_{k_{\nu}}-\alpha_{j}\right)$ and summing with respect to $j$ we obtain the relation

$$
\sum_{j=1}^{n} \frac{a_{j}(x) f_{j}(x) v_{k_{\nu}}(x)}{\lambda_{k_{\nu}}-\alpha_{j}}=\sum_{j=1}^{n} \sum_{\substack{l=-\infty \\ \mu=1,2, \ldots, n}}^{+\infty} c_{l_{\mu}} \frac{a_{j}(x) u_{l_{\mu}}(x) v_{k_{\nu}}(x)}{\left(\lambda_{k_{\nu}}-\alpha_{j}\right)\left(\lambda_{l_{\mu}}-\alpha_{j}\right)} .
$$

On the assumption that the series on the right of this equation converge and may be integrated term by term between the points $a$ and $b$ it is found in view of relation (5) and the fact that $u_{k_{\nu}}(x) v_{k_{\nu}}(x) \equiv 1$, that

$$
\int_{a}^{b} \sum_{j=1}^{n} \frac{a_{j}(x) f_{j}(x) v_{k_{\nu}}(x)}{\left(\lambda_{k_{\nu}}-\alpha_{j}\right)} d x=c_{k_{\nu}} \sum_{j=1}^{n} \frac{A_{j}(b)}{\left(\lambda_{k_{\nu}}-\alpha_{j}\right)^{2}} .
$$

Inasmuch as the sum on the right was assumed to be different from zero,

$$
c_{k_{\nu}}=\frac{\int_{a}^{b} \sum_{j=1}^{n} \frac{a_{j}(x) f_{j}(x) v_{k_{\nu}}(x)}{\lambda_{k_{\nu}}-\alpha_{j}} d x}{\sum_{j=1}^{n} \frac{A_{j}(b)}{\left(\lambda_{k_{\nu}}-\alpha_{j}\right)^{2}}},
$$

and (6) is more explicitly

$$
f_{i}(x)=\sum_{\substack{k=-\infty \\ \nu=1,2, \ldots, n}}^{+\infty} \int_{a}^{b} \sum_{j=1}^{n} \frac{a_{j}(t) f_{j}(t) v_{k_{\nu}}(t) u_{k_{\nu}}(x)}{\left(\lambda_{k_{\nu}}-\alpha_{j}\right)\left(\lambda_{k_{\nu}}-\alpha_{i}\right) \sum_{j=1}^{n} \frac{A_{j}(b)}{\left(\lambda_{k_{\nu}}-\alpha_{j}\right)^{2}}} \cdot d t .
$$

Instead of returning to a rigorous establishment of this formal result we shall investigate directly the questions, does the series in (8) converge? and if so, what does it represent? Before proceeding to this, however, it is of interest to observe the nature of the reduction of expansion (8) in a particularly simple case, namely that in which the coefficients $a_{j}(x)$ are all constants. For simplicity let $n$ be chosen as $n=2$.

From (3) the characteristic values $\lambda_{k_{1}}$ and $\lambda_{k_{2}}$ are the roots of the equation

$$
\frac{a_{1}}{\lambda-\alpha_{1}}+\frac{a_{2}}{\lambda-\alpha_{2}}=\frac{2 k \pi i}{(b-a)}
$$

and since, therefore,

$$
\frac{a_{1}}{\lambda_{k_{1}}-\alpha_{1}}+\frac{a_{2}}{\lambda_{k_{1}}-\alpha_{2}}=\frac{a_{1}}{\lambda_{k_{2}}-\alpha_{1}}+\frac{a_{2}}{\lambda_{k_{2}}-\alpha_{2}},
$$


it is readily seen that

$$
a_{2}\left(\frac{\lambda_{k_{1}}-\alpha_{1}}{\lambda_{k_{1}}-\alpha_{2}}\right)=-a_{1}\left(\frac{\dot{\lambda}_{k_{2}}-\alpha_{2}}{\lambda_{k_{2}}-\alpha_{1}}\right)
$$

Furthermore, since $a_{1}$ and $a_{2}$ are constants,

$$
u_{k_{1}}(x) \equiv u_{k_{2}}(x) \equiv e^{2 k \pi i \frac{x-a}{b-a}}
$$

The expansion (8) for $f_{1}(x)$ is, therefore,

$$
\begin{aligned}
f_{1}(x)=\sum_{k=-\infty}^{+\infty} \int_{a}^{b}\left\{\frac{\frac{a_{1} f_{1}(t)}{\left(\lambda_{k_{1}}-\alpha_{1}\right)^{2}}+\frac{a_{2} f_{2}(t)}{\left(\lambda_{k_{1}}-\alpha_{2}\right)\left(\lambda_{k_{1}}-\alpha_{1}\right)}}{\left[\frac{a_{1}}{\left(\lambda_{k_{1}}-\alpha_{1}\right)^{2}}+\frac{a_{2}}{\left(\lambda_{k_{1}}-\alpha_{2}\right)^{2}}\right](b-a)}\right. \\
\left.\quad+\frac{\frac{a_{1} f_{1}(t)}{\left(\lambda_{k_{2}}-\alpha_{1}\right)^{2}}+\frac{a_{2} f_{2}(t)}{\left(\lambda_{k_{2}}-\alpha_{2}\right)\left(\lambda_{k_{2}}-\alpha_{1}\right)}}{\left[\frac{a_{1}}{\left(\lambda_{k_{2}}-\alpha_{1}\right)^{2}}+\frac{a_{2}}{\left(\lambda_{k_{2}}-\alpha_{2}\right)^{2}}\right](b-a)}\right\} v_{k}(t) \iota_{k}(x) a t .
\end{aligned}
$$

Multiplying both numerator and denominator of each fraction of the integrand by a suitable factor, the expression within the brace takes the form

$$
\left\{\frac{f_{1}(t)+f_{2}(t) \frac{a_{2}}{a_{1}}\left(\frac{\lambda_{k_{1}}-\alpha_{1}}{\lambda_{k_{1}}-\alpha_{2}}\right)}{1+\frac{a_{2}}{a_{1}}\left(\frac{\lambda_{k_{1}}-\alpha_{1}}{\lambda_{k_{1}}-\alpha_{2}}\right)^{2}}+\frac{f_{1}(t) \frac{a_{1}}{a_{2}}\left(\frac{\lambda_{k_{2}}-\alpha_{2}}{\lambda_{k_{2}}-\alpha_{1}}\right)^{2}+f_{2}(t) \frac{\lambda_{k_{2}}-\alpha_{2}}{\lambda_{k_{2}}-\alpha_{1}}}{\frac{a_{1}}{a_{2}}\left(\frac{\lambda_{k_{2}}-\alpha_{2}}{\lambda_{k_{2}}-\alpha_{1}}\right)^{2}+1}\right\}
$$

This reduces on account of (9) to

$$
\left\{\frac{f_{1}(t)-f_{2}(t) \frac{\lambda_{k_{2}}-\alpha_{2}}{\lambda_{k_{3}}-\alpha_{1}}+f_{1}(t) \frac{a_{1}}{a_{2}}\left(\frac{\lambda_{k_{2}}-\alpha_{2}}{\lambda_{k_{1}}-\alpha_{1}}\right)^{2}+f_{2}(t) \frac{\lambda_{k_{2}}-\alpha_{2}}{\lambda_{k_{2}}-\alpha_{1}}}{1+\frac{a_{1}}{a_{2}}\left(\frac{\lambda_{k_{2}}-\alpha_{2}}{\lambda_{k_{2}}-\alpha_{1}}\right)^{2}}\right\}
$$

namely to $f_{1}(t)$, and the expansion in question may be written

$$
f_{1}(x)=\sum_{k=-\infty}^{+\infty} \frac{1}{b-a} \int_{a}^{b} f_{1}(t) e^{2 k \pi i \frac{x-t}{b-a}} d t
$$


Inasmuch as the imaginary terms of the series for the values of $k$ numerically equal but of opposite sign cancel, if $f_{1}(x)$ is real, the series for such a function reduces to

$$
f_{1}(x)=\sum_{k=-\infty}^{+\infty} \frac{1}{b-a} \int_{a}^{b} f_{1}(t) \cos \frac{2 k \pi}{b-a}(x-t) d t .
$$

Hence expansion (8) for $f_{1}(x)$ is none other than its Fourier's series, and in precisely similar manner the reduction of (8) to the Fourier's series in the case of $f_{\mathbf{z}}(x)$ may be shown. The generalization is, therefore, one from constant coefficients $a_{j}$ to variable coefficients $a_{j}(x)$.

\section{THE EXPANSION AS A CONTOUR INTEGRAL}

When $\lambda$ is not a characteristic value of system (2) there exists a unique Green's function, $G(x, t, \lambda)$, which is characterized by the fact that the solution of the non-homogeneous system

$$
\begin{aligned}
& y^{\prime}(x)=\sum_{j=1}^{n} \frac{a_{j}(x)}{\lambda-\alpha_{j}} y(x)+\omega(x), \\
& y(a)=y(b),
\end{aligned}
$$

is given by the formula

$$
y(x)=\int_{a}^{b} G(x, t, \lambda) \omega(t) d t . *
$$

The explicit formula for $G(x, t, \lambda)$ is in this case

$$
G(x, t, \lambda)=\frac{u(x, \lambda) v(t, \lambda)}{2}\left[ \pm 1+\frac{1+u(b, \lambda)}{1-u(b, \lambda)}\right],
$$

where the ambiguous sign must be chosen

and where

$$
+ \text { for } t<x, \quad-\text { for } t>x,
$$

while

$$
u(x, \lambda)=e^{\sum_{=1}^{n} \frac{A_{j}(x)}{\lambda-a_{j}}}
$$

$$
u(x, \lambda) v(x, \lambda) \equiv 1 \text {. }
$$

* Bôcher, Bulletin of the American Mathematical Society. vol. 7 (1901), p. 297. 
It is seen, therefore, that $G(x, t, \lambda)$ considered as a function of the parameter $\lambda$ is analytic over the entire plane except for a pole at each of the characteristic values, these poles being of the first order when the characteristic equations have only simple roots. The residue at $\lambda=\lambda_{k \nu}$ is, of course, given by the formula

or, since

$$
R_{k_{\nu}}(x, t)=\frac{u\left(x, \lambda_{k_{\nu}}\right) v\left(t, \lambda_{k_{\nu}}\right)}{2} \frac{1+u\left(b, \lambda_{k_{\nu}}\right)}{\frac{d}{d \lambda}[1-u(b, \lambda)]_{\lambda=\lambda_{k_{\nu}}}}
$$

more explicitly by

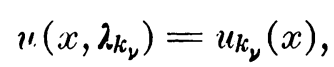

$$
R_{k_{\nu}}(x, t)=\frac{u_{k_{\nu}}(x) v_{k_{\nu}}(t)}{\sum_{j=1}^{n} \frac{A_{j}(b)}{\left(\lambda_{k_{\nu}}-\alpha_{j}\right)^{2}}}
$$

Hence if $\gamma_{k_{\nu}}$ is any closed contour in the $\lambda$ plane which includes $\lambda_{k_{\nu}}$ and no other characteristic values, and none of points $\alpha_{i}(i=1,2, \cdots, n)$, then

$$
\frac{1}{2 \pi i} \int_{\gamma_{k_{\nu}}} \frac{G(x, t, \lambda)}{\left(\lambda-\alpha_{j}\right)\left(\lambda-\alpha_{i}\right)} d \lambda=\frac{v_{k_{\nu}}(t) u_{k_{\nu}}(x)}{\left(\lambda_{k_{\nu}}-\alpha_{j}\right)\left(\lambda_{k_{\nu}}-\alpha_{i}\right) \sum_{j=1}^{n} \frac{A_{j}(b)}{\left(\lambda_{k_{\nu}}-\alpha_{j}\right)^{2}}} .
$$

Multiplying this by $a_{j}(t) f_{j}(t)$, summing with respect to $j$, and integrating with respect to $t$, we obtain the relation

$$
\begin{aligned}
\frac{1}{2 \pi i} \int_{\gamma_{k_{\nu}}} \int_{a}^{b} \sum_{j=1}^{n} \frac{a_{j}(t) f_{j}(t) G(x, t, \lambda)}{\left(\lambda-\alpha_{j}\right)(\lambda-}-\frac{\left.\alpha_{i}\right)}{(\lambda)} d t d \lambda & \\
& =\int_{a}^{b} \sum_{j=1}^{n} \frac{a_{j}(t) f_{j}(t) v_{k_{\nu}}(t) u_{k_{\nu}}(x)}{\left(\lambda_{k_{\nu}}-\alpha_{j}\right)\left(\lambda_{k_{\nu}}-\alpha_{i}\right) \sum_{j=1}^{n} \frac{A_{j}(b)}{\left(\lambda_{k_{\nu}}-\alpha_{j}\right)^{2}}} d t .
\end{aligned}
$$

The right-hand side of this equation is, however, precisely the $k_{\nu}$ th term of the series in (8)* from which it follows that if $\gamma_{m}$ is a contour enclosing the

${ }^{*}$ If a number of characteristic values coincide and are located at $\lambda_{k_{\nu}}$ the corresponding terms of series (8) taken together are to be replaced by

$$
\int_{a}^{b} \sum_{j=1}^{n} \frac{a_{j}(t) f_{j}(t) R_{k_{\nu}}(x, t)}{\left(\lambda_{k_{\nu}}-a_{j}\right)\left(\lambda_{k_{\nu}}-a_{i}\right)} d t
$$

where $R_{k y}(x, t)$ is the residue of $G(x, t, \lambda)$ at $\lambda=\lambda_{k y}$. 
characteristic values $\lambda_{1}, \lambda_{2}, \cdots, \lambda_{m}$, and no others, then $S_{m}^{(i)}(x)$, the sum of the terms of series (8) corresponding to $\lambda_{1}, \lambda_{2}, \cdots, \lambda_{m}$ is given by

$$
S_{m}^{(i)}(x)=\frac{1}{2 \pi i} \int_{\gamma_{m}} \int_{a}^{b} \sum_{j=1}^{n} \frac{a_{j}(t) f_{j}(t) G(x, t, \lambda)}{\left(\lambda-\alpha_{j}\right)\left(\lambda-\alpha_{i}\right)} d t d \lambda .
$$

IV. CONVERGENCE OF THE SERIES

The equation for the characteristic values has already been given, (3), and it was observed that these values cluster about the $n$ points $\alpha_{1}, \alpha_{2}, \cdots, \alpha_{n}$, for $|k|$ large. The substitution

$$
\frac{1}{\lambda-\alpha_{\tau}}=\varrho
$$

projects the point $\alpha_{\tau}$ to $\infty$ and transforms (3) into

$$
\sum_{j=1}^{n} \frac{A_{j}(b)}{\alpha_{\tau}-\alpha_{j}+1 / \varrho}=2 k \pi i
$$

which may also be written

$$
\varrho_{k_{\tau}}=\frac{1}{A_{\tau}(b)}\left\{2 k \pi i+\sum_{\substack{j=1 \\ j \neq \tau}}^{n}\left[\frac{A_{j}(b)}{\alpha_{j}-\alpha_{\tau}}+1 / \varrho \frac{A_{j}(b)}{\left(\alpha_{\tau}-\alpha_{j}\right)\left(\alpha_{\tau}-\alpha_{j}+1 / \varrho\right)}\right]\right\} .
$$

From this every characteristic value is seen to be of the form

$$
\varrho_{k_{\tau}}=\frac{2 k \pi i+C}{A_{\tau}(b)}+\frac{\sigma(\varrho)}{\varrho},
$$

$C$ being a constant and $\sigma(\varrho)$ being bounded for $|\varrho|>R$.

Consider a small circle of fixed radius $\varepsilon$ drawn about the points $\varrho=\frac{2 k \pi i+C}{A_{\tau}(b)}$, where the quantity $C$ is that of formula (14). For $|\varrho|$ sufficiently large the point

$$
\frac{2 k \pi i+C}{A_{\tau}(b)}+\frac{\sigma(\varrho)}{\varrho}
$$

will lie within this circle for any $\varrho$ on the circumference, and hence, as $\varrho$ describes this circumference.

$$
\operatorname{arc}\left\{\varrho-\left(\frac{2 k \pi i+C}{A_{\tau}(b)}+\frac{\sigma(\varrho)}{\varrho}\right)\right\}
$$


increases by $2 \pi$. It follows, therefore, that for $|\varrho|>R$ the characteristic values all lie within these small circles, and that each circle contains just one characteristic value.

It is clear from this that the interval between any two characteristic values given by (14) for consecutive values of $k$ becomes more and more nearly equal as $|k|$ is taken larger and larger, and that it is possible, therefore, to choose a set of circles $\bar{C}_{j}$ with centers in $\varrho=0$ and of successively larger radii (there being always one whose radius exceeds any given constant) which are such that the distance between any point of a circle $\bar{C}_{j}$ and any characteristic value exceeds some constant $\delta>0$. Let $C_{j}$ be used now to denote any circle of the set into which the $\overrightarrow{C_{j}}$ 's are transformed by the inverse of substitution (12). Having defined the set $C_{j}$ in this manner let the procedure be repeated for each of the remaining points $\alpha_{1}, \cdots, \alpha_{n}$, and the $n$ sets of circles $C_{1}, C_{2}, \cdots, C_{n}$ be thus determined. It can easily be shown that there exists a positive lower limit $M$ for the values of

$$
|1-u(b, \lambda)|, \text { and }|v(b, \lambda)-1|
$$

when $\lambda$ lies on any of the circles $C_{i}(i=1,2, \cdots, n)$.

Returning now to formula (11), let the contour $\gamma_{m}$ be composed of circles $C$ chosen in the manner described above, one such circle being drawn about each of the points $\alpha_{1}, \alpha_{2}, \cdots, \alpha_{n}$, and described in the negative sense. Then $S_{m}^{(i)}(x)$, as given by the formula

$$
S_{m}^{(i)}(x)=\frac{1}{2 \pi i} \int_{-C_{1}-C_{2}-\ldots-C_{n}} \int_{a}^{b} \sum_{j=1}^{n} \frac{a_{j}(t) f_{j}(t) G(x, t, \lambda)}{\left(\lambda-\alpha_{j}\right)\left(\lambda-\alpha_{i}\right)} d t d \lambda,
$$

will represent the sum of the terms corresponding to the characteristic values excluded by all the circles in question. As the circles are taken smaller and smaller, (15) will sum a larger and larger number of terms, and the limit of the integral as the process of successively replacing the circles $C$ by smaller circles $C$ is allowed to continue indefinitely will, if it exists, represent the sum of the series. We proceed to the evaluation of this limit.

Upon writing (15) in the form

$$
S_{m}^{(i)}(x)=\sum_{l=1}^{n} \frac{1}{2 \pi i} \int_{-C_{l}} \int_{a}^{b} \frac{G(x, t, \lambda)}{\lambda-\alpha_{i}} \sum_{j=1}^{n} \frac{a_{j}(t) f_{j}(t)}{\lambda-\alpha_{j}} d t d \lambda,
$$

we have in the sum on the right integrals of two types, namely those in which $j$ and $l$ are not both equal to $i$, and that for which $j, l$, and $i$ are all 
equal. In the evaluation of these integrals it will be convenient to refer to the fact established by

LEMMA I. Given $\varphi(t, \varrho)$ any finction such that (i) $|\varphi(t, \varrho)|<K$ for $\alpha \leqq t$ $\leqq \beta, \gamma \leqq \theta \leqq \delta,|\varrho|>R$, and (ii) $\lim _{|\rho| \rightarrow \infty}|\varphi(t, \varrho)|=0$ uniformly for $\alpha+\varepsilon$ $\leqq t \leqq \beta-\varepsilon, \gamma+\varepsilon \leqq \theta \leqq \delta-\varepsilon, \varepsilon$ being any sufficiently small positive constant, and $\theta$ denoting arc $\varrho$. Then if

$$
I=\frac{1}{2 \pi i} \int_{C} \int_{a}^{\beta} \varphi(t, \varrho) d t \frac{d \varrho}{\varrho},
$$

$C$ being that arc of circle $|\varrho|=r$ drawn from the point $\theta=\gamma$ to the point $\boldsymbol{\theta}=\boldsymbol{\delta}$, it follows that

$$
\lim _{\rho \rightarrow \infty} I=0
$$

PR00F. Setting $\varrho=r e^{i \theta}$, and defining the function $\psi$ by the relation $\varphi(t, \varrho)=\psi(t, \theta, r)$ we have

whence

$$
I=\frac{1}{2 \pi} \int_{\gamma}^{\delta} \int_{\alpha}^{\beta} \psi(t, \theta, r) d t d \theta
$$

$$
\begin{aligned}
|I| \leqq \frac{1}{2 \pi}\left\{\left|\int_{\gamma+\varepsilon}^{\delta-\varepsilon} \int_{\alpha}^{\beta-\varepsilon} \psi(t, \theta, r) d t d \theta\right|\right. & +\int_{\alpha}^{\beta \gamma} \int_{\gamma}^{\gamma+\varepsilon} K d \theta d t+\int_{\alpha}^{\beta} \int_{\delta-\varepsilon}^{\delta} K d \theta d t \\
& +\int_{\gamma+\varepsilon}^{\delta} \int_{a}^{\alpha+\varepsilon} K d t d \theta+\int_{\gamma+\varepsilon}^{\delta-\varepsilon} \int_{\beta-\varepsilon}^{\beta} K d t d \theta \mid
\end{aligned}
$$

From this it is apparent, however, inasmuch as the limit of the first integral on the right is zero, that for $r$ sufficiently large

$$
|I|<\frac{2 l K}{\pi} \varepsilon, \quad \text { wherc }\left\{\begin{array}{l}
l \geqq|\boldsymbol{\beta}-\alpha|, \\
l \geqq|\delta-\gamma| .
\end{array}\right.
$$

Since $\varepsilon$ was arbitrary, however, this implies that

$$
\lim _{\rho \rightarrow \infty} I=0
$$

Let us consider now the integrals in formula (15a), assuming the functions $f_{j}(x)$ which we wish to develop to consist, in the interval $a \leqq x \leqq b$, of at 
most a finite number of pieces, each continuous and having a continuous first derivative. We have

LEMMA II. If

$$
I_{2}=\frac{1}{2 \pi i} \int_{-C_{\tau}} \int_{a}^{b} \frac{G(x, t, \lambda) a_{\mu}(t) f_{\mu}(t)}{\left(\lambda-\alpha_{\nu}\right)\left(\lambda-\alpha_{\mu}\right)} d t d \lambda
$$

where

$$
(\tau, \mu) \neq(\nu, \nu),
$$

then

$$
\lim I_{2}=0,
$$

when $C_{\tau}$ is taken successively as a smaller and smaller circle of the type $C$ defined above, drawn about the point $\alpha_{\tau}$.

Proof. Transforming the plane of the parameter by the substitution (12) we have

$$
I_{2}=\frac{-1}{2 \pi i} \int_{\bar{c}_{\tau}} \int_{a}^{b} \Phi(t, \varrho) G\left(x, t, \alpha_{\tau}+1 / \varrho\right) d t \frac{d \varrho}{\varrho}
$$

where $\boldsymbol{\Phi}(t, \varrho)$, being given by the formula

$$
\Phi(t, \varrho)=\frac{a_{\mu}(t) f_{\mu}(t)}{\varrho\left(\alpha_{\tau}-\alpha_{\nu}+1 / \varrho\right)\left(\alpha_{\tau}-\alpha_{\mu}+1 / \varrho\right)},
$$

is seen to be bounded for $|\varrho|>R$, since $a_{\mu}(t)$ and $f_{\mu}(t)$ are bounded, while at least one of the quantities $\alpha_{\nu}$ and $\alpha_{\mu}$ differs from $\alpha_{\tau} . \bar{C}_{\tau}$ is now a circle $|\varrho|=r$. The expression for the Green's function, $G\left(x, t, \alpha_{\tau}+1 / \varrho\right)$, is given in (10) but may be more conveniently used in the following equivalent forms:

when $t<x$, and

$$
G\left(x, t, \alpha_{\tau}+1 / \varrho\right)=\frac{u(x, \varrho) v(t, \varrho)}{1-u(b, \varrho)}=\frac{u(x, \varrho) v(t, \varrho) v(b, \varrho)}{v(b, \varrho)-1}
$$

$$
G\left(x, t, \alpha_{-}+1 / \varrho\right)=\frac{u(x, \varrho) v(t, \varrho) u(b, \varrho)}{1-u(b, \varrho)}=\frac{u(x, \varrho) v(t, \varrho)}{v(b, \varrho)-1}
$$

when $t>x$.

Denoting by $\overline{C_{\tau}^{\prime}}$ the arc of $\overline{C_{\tau}^{\prime}}$ which lies in the half-plane $R\left\{\rho a_{\tau}(t)\right\} \geqq 0$,* and by $\bar{C}_{\tau}^{\prime \prime}$ the arc on which $R\left\{\rho a_{\tau}(t)\right\}<0, I_{2}$ may be more explicitly written as the sum of the integrals

* The notation $R\{\omega\}$ is used to indicate "the real part of $\omega$ ". 


$$
\begin{aligned}
& I_{21}=\frac{-1}{2 \pi i} \int_{\bar{C}_{\tau}^{\prime}} \int_{a}^{x} \Phi(t, \varrho) \frac{u(x, \varrho) v(t, \varrho) v(b, \varrho)}{v(b, \varrho)-1} d t \frac{d \varrho}{\varrho}, \\
& I_{22}=\frac{-1}{2 \pi i} \int_{\bar{C}_{\tau}^{\prime \prime}} \int_{a}^{x} \Phi(t, \varrho) \frac{u(x, \varrho) v(t, \varrho)}{1-u(b, \varrho)} d t \frac{d \varrho}{\varrho}, \\
& I_{23}=\frac{-1}{2 \pi i} \int_{\bar{C}_{\tau}^{\prime}} \int_{x}^{b} \Phi(t, \varrho) \frac{u(x, \varrho) v(t, \varrho)}{v(b, \varrho)-1} d t \frac{d \varrho}{\varrho}, \\
& I_{24}=\frac{-1}{2 \pi i} \int_{\bar{C}_{t}^{\prime \prime}} \int_{x}^{b} \Phi(t, \varrho) \frac{u(x, \varrho) v(t, \varrho) u(b, \varrho)}{1-u(b, \varrho)} d t \frac{d \varrho}{\varrho} .
\end{aligned}
$$

The notation $u(x, \varrho)$ has been used here in preference to the more clumsy $u\left(x, \alpha_{\tau}+1 / \varrho\right)$.

Let us consider the integrand of $I_{21}$ which is typical. For $\varrho$ on $\overline{C_{\tau}^{\prime}}$ the factor $\frac{\Phi(t, \varrho)}{v(b, \varrho)-1}$ is bounded, while the remaining factor is

$$
e^{\rho\left\{A_{\tau}(x)-A_{\tau}(t)-A_{\tau}(b)\right\}}+\sum_{\substack{j=1 \\ j \neq \tau}}^{n} \frac{A_{j}(x)-A_{j}(t)-A_{j}(b)}{a_{\tau}-a_{j}+1 / \rho}
$$

If $x \neq b$ this clearly approaches the limit zero uniformly as $|\varrho|$ increases, for all values of $t$ in the interval $a \leq t \leq x$ and $\varrho$ in any closed sector within and on the boundary of which $R\left\{\varrho a_{\tau}(t)\right\}>0$. The same is true also if $a \leqq x \leqq b$ and $a+\varepsilon \leqq t \leqq x$, while the value of the expression for $a \leqq x \leqq b$, and all values of $t$ in the interval $a \leqq t \leqq x$ and $R\left\{\rho a_{\tau}(t)\right\} \geqq 0$ is bounded. The entire integrand, is, therefore, for any given $x$, of the type $\varphi(t, \varrho)$ of LemmaI, and accordingly

$$
\lim _{|\rho| \rightarrow \infty} I_{21}=0
$$

Inasmuch as the same reasoning may be applied to each of the remaining integrals $I_{2 i}$, it is readily found that

$$
\lim _{|\rho| \rightarrow \infty} I_{2}=0
$$


The integral in (15a) which results from $i=l=j$ remains to be considered. We have

LEMMA III. If

$$
I_{3}=\frac{1}{2 \pi i} \int_{-C_{\tau}} \int_{a}^{b} \frac{G(x, t, \lambda) a_{\tau}(t) f_{\tau}(t)}{\left(\lambda-\alpha_{\tau}\right)^{2}} d t d \lambda
$$

then

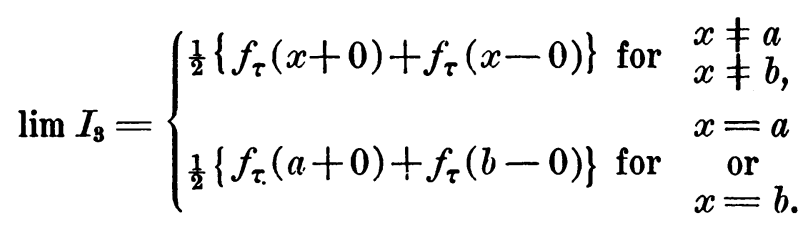

Proof. Applying again the transformation (12) we obtain the form

$$
I_{3}=\frac{-1}{2 \pi i} \int_{C_{\tau}} \int_{a}^{b} G\left(x, t, \alpha_{\tau}+1 / \varrho\right) a_{\tau}(t) f_{\tau}(t) d \varrho,
$$

or, substituting the values of $G\left(x, t, \alpha_{\tau}+1 / \varrho\right)$, as given by formula (10),

$$
\begin{aligned}
I_{3}=\frac{-1}{2 \pi i} \int_{\bar{C}_{\tau}} \frac{u(x, \varrho)}{1-u(b, \varrho)}\left\{\int_{a}^{x} v(t, \varrho) a_{\tau}(t) f_{\tau}(t) d t\right. & \\
& \left.+u(b, \varrho) \int_{x}^{b} v(t, \varrho) a_{\tau}(t) f_{\tau}(t) d t\right\} d \varrho .
\end{aligned}
$$

The integration by parts indicated by the relation

$$
\begin{aligned}
&-\int_{\gamma}^{\delta} a_{\tau}(t) f_{\tau}(t) v(t, \varrho) d t=1 / \varrho\left\{f_{\tau}(\delta-0) v(\delta, \varrho)\right. \\
&\left.-f_{\tau}(\gamma+0) v(\gamma, \varrho)-\int_{\gamma}^{\delta}\left\{f_{\tau}^{\prime}(t)-f_{\tau}(t) \sum_{\substack{j=1 \\
j \neq \tau}}^{n} \frac{a_{j}(t)}{\alpha_{\tau}-\alpha_{j}+1 / \varrho}\right\} v(t, \varrho) d t\right\}
\end{aligned}
$$

now yields a form for $I_{3}$, of which the various terms may be collected into the following expressions:

$$
I_{31}=\frac{-1}{2 \pi i} \int_{\bar{C}_{\tau}} \int_{a}^{b}\left\{f_{\tau}^{\prime}(t)-f_{\tau}(t) \sum_{\substack{j=1 \\ j \neq \tau}}^{n} \frac{a_{j}(t)}{\alpha_{\tau}-\alpha_{j}+1 / \varrho}\right\} G\left(x, t, \alpha_{\tau}+1 / \varrho\right) d t \frac{d \varrho}{\varrho},
$$




$$
I_{32}=\left\{\begin{array}{l}
\frac{1}{2 \pi i} \int_{\bar{C}_{\tau}}\left[\left(f_{\tau}(x-0)-\left[f_{\tau}(a+0)-f_{\tau}(b-0)\right] u(x, \varrho)\right.\right. \\
\text { when } \left.\left.x \neq a, x \neq b, \quad-f_{\tau}(x+0) u(b, \varrho)\right) \frac{1}{1-u(b, \varrho)}\right] \frac{d \varrho}{\varrho} \\
\frac{1}{2 \pi i} \int_{\bar{C}_{\tau}} \frac{f_{\tau}(b-0)-f_{\tau}(a+0) u(b, \varrho)}{1-u(b, \varrho)} \frac{d \varrho}{\varrho} \\
\text { when } x=a \text { or } x=b .
\end{array}\right.
$$

Now inasmuch as

$$
\left\{f_{\tau}^{\prime}(t)-f_{\tau}(t) \sum_{\substack{j=1 \\ j \neq \tau}}^{n} \frac{a_{j}(t)}{\alpha_{\tau}-\alpha_{j}+1 / \varrho}\right\}
$$

is bounded for $|\varrho|>R$, the integral $I_{31}$ is seen to be of precisely the same type as the integral $I_{2}$ in formula (16), and it follows accordingly that

$$
\lim I_{31}=0 \text {. }
$$

Further, from the more explicit forms

$$
\begin{gathered}
I_{32}=\frac{1}{2 \pi i} \int_{\overline{\bar{C}}_{\tau}^{\prime}}^{\partial}\left\{\frac{\left[f_{\tau}(x-0)-f_{\tau}(x+0)\right]-\left[f_{\tau}(a+0)-f_{\tau}(b-0)\right] u(x, \varrho)}{1-u(b, \varrho)}\right. \\
\left.\quad+f_{\tau}(x+0)\right\} \frac{d \varrho}{\varrho}+\frac{1}{2 \pi i} \int_{\bar{C}_{\tau}^{\prime \prime}}\left\{f_{\tau}(x-0)\right. \\
\left.+\frac{\left[f_{\tau}(x-0)-f_{\tau}(x+0)\right] u(b, \varrho)-\left[f_{\tau}(a+0)-f_{\tau}(b-0)\right] u(x, \varrho)}{1-u(b, \varrho)}\right\} \frac{d \varrho}{\varrho}
\end{gathered}
$$

when $x \neq a, x \neq b$,

$$
\begin{aligned}
I_{32}=\frac{1}{2 \pi i} \int_{\tilde{C}_{\tau}^{\prime}}\left\{\frac{\left[f_{\tau}(b-0)-f_{\tau}(a+0)\right]}{1-u(b, \varrho)}+f_{\tau}(a+0)\right\} \frac{d \varrho}{\varrho} \\
\quad+\frac{1}{2 \pi i} \int_{\bar{c}_{\tau}^{\prime \prime}}\left\{f_{\tau}(b-0)+\frac{\left[f_{\tau}(b-0)-f_{\tau}(a+0)\right] u(b, \varrho)}{1-u(b, \varrho)}\right\} \frac{d \varrho}{\varrho}
\end{aligned}
$$


when $x=a$ or $x=b$, the evaluation of $I_{32}$ is simple. The fractional parts of these integrands are functions of such type that their integrals over the respective paths of integration vanish when the limit as $|\varrho|=\infty$ is taken, as may be determined by an argument analogous to that used in the proof of Lemma I. It follows, therefore, that

$$
\lim _{|\rho| \rightarrow \infty} I_{32}=\frac{1}{2}\left\{f_{\tau}(x+0)+f_{\tau}(x-0)\right\}
$$

when $x \neq a, x \neq b$,

$$
\lim _{|\rho| \rightarrow \infty} I_{32}=\frac{1}{2}\left\{f_{\tau}(a+0)+f_{\tau}(b-0)\right\},
$$

when $x=a$ or $x=b$. This establishes the final lemma.

THEOREM. Given any $n$ functions $f_{1}(x), f_{2}(x), \cdots, f_{n}(x)$, which, in the interval $a \leqq x \leqq b$, consist of at most a finite number of pieces, each real and continuous and having a continuous derivative; then these functions may be simultaneously expanded in series of the form

$$
f_{i}(x)=\sum_{\substack{k=-\infty \\ j=1,2, \ldots, n .}}^{+\infty} C_{k j} \frac{u_{k_{j}}(x)}{\lambda_{k_{j}}-\alpha_{i}} \quad(i=1,2, \cdots, n)
$$

In this the $\lambda$ 's and $u$ 's are respectively the characteristic values and functions of a differential system

$$
\begin{aligned}
u^{\prime}(x) & =\sum_{j=1}^{n} \frac{a_{j}(x)}{\lambda-\alpha_{j}} u(x), \\
u(a) & =u(b)
\end{aligned}
$$

each $\alpha_{i}$ being a constant, while each $a_{j}(x)$ is real, maintains the same sign, and is bounded and integrable throughout the interval $a \leqq x \leqq b$. This expansion for $f_{i}(x)$ will, moreover, converge to $\frac{1}{2}\left\{f_{i}(x+0)+\overline{f_{i}}(x-0)\right\}$ for any interior point of the interval, and to $\frac{1}{2}\left\{f_{i}(a+0)+f_{i}(b-0)\right\}$ at the end points.

PR00F. From the choice of notation $f_{i}(x)=\lim _{m \rightarrow \infty} S_{m}^{(i)}(x)$, and hence by $(15 a)$

$$
f_{i}(x)=\lim \sum_{j, \tau=1}^{n} \frac{1}{2 \pi i} \int_{-C_{\tau}} \int_{a}^{b} \frac{a_{j}(t) f_{j}(t) G(x, t, \lambda)}{\left(\lambda-\alpha_{j}\right)\left(\lambda-\alpha_{i}\right)} d t d \lambda .
$$


The evaluation of this expression may be simplified by writing it in the form

$$
\begin{aligned}
f_{i}(x)= & \lim \sum_{\substack{j, \tau=1 \\
(j, \tau) \neq(i, i)}}^{n} \frac{1}{2 \pi i} \int_{-C_{\tau}} \int_{a}^{b} \frac{a_{j}(t) f_{j}(t) G(x, t, \lambda)}{\left(\lambda-\alpha_{j}\right)\left(\lambda-\alpha_{i}\right)} d t d \lambda \\
& \quad+\lim \frac{1}{2 \pi i} \int_{-C_{i}} \int_{a}^{b} \frac{a_{i}(t) f_{i}(t) G(x, t, \lambda)}{\left(\lambda-\alpha_{i}\right)^{2}} d t d \lambda,
\end{aligned}
$$

for the first of these limits vanishes by Lemma II. Hence the expansion for $f_{i}(x)$ converges to the value of the final limit above, and this is by Lemma III

$$
\begin{aligned}
& \frac{1}{2}\left\{f_{i}(x+0)+f_{i}(x-0)\right\}, \text { when } x \neq a, x \neq b, \\
& \frac{1}{2}\left\{f_{i}(a+0)+f_{i}(b-0)\right\}, \text { when } x=a, x=b,
\end{aligned}
$$

Q.E.D.

\section{HARVARD UNIVERSITY,}

Cambridge, Mass. 